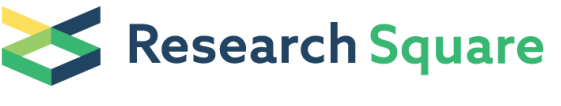 \\ Preprints are preliminary reports that have not undergone peer review. \\ They should not be considered conclusive, used to inform clinical practice, or referenced by the media as validated information. \\ Reconstructed Brain Like Neural Network R-KFDNN \\ zhu rongrong ( $\nabla$ rongrongzhu1969@163.com ) \\ Fudan University https://orcid.org/0000-0003-0385-4506
}

\section{Article}

Keywords: Brain like, Nervous system injury and repair, Flexible neural network, Key group generation sequence, High-dimensional information field, Memory analysis

Posted Date: December 27th, 2021

DOI: https://doi.org/10.21203/rs.3.rs-1158026/v2

License: (c) (1) This work is licensed under a Creative Commons Attribution 4.0 International License. Read Full License 


\title{
Reconstructed Brain Like Neural Network R-KFDNN
}

\author{
Zhu Rongrong, Fudan University, Shanghai, China \\ rongrongzhu1969@163.com
}

\begin{abstract}
Through the neural system damage and repair process of human brain, we can construct the complex deep learning and training of the repair process such as the damage of brain like high-dimensional flexible neural network system or the local loss of data, so as to prevent the dimensional disaster caused by the local loss of high-dimensional data. How to recover and extract feature information when the damaged neural system (flexible neural network) has amnesia or local loss of stored information. Information extraction generally exists in the distribution table of the generation sequence of the key group of the higher dimension or the lower dimension to find the core data stored in the brain. The generation sequence of key group exists in a hidden time tangent cluster. Brain like slice data processing runs on different levels, different dimensions, different tangent clusters and cotangent clusters. The key group in the brain can be regarded as the distribution table of memory fragments. Memory parsing has mirror reflection and is accompanied by the loss of local random data. In the compact compressed time tangent cluster, it freely switches to the high-dimensional information field, and the parsed key is buried in the information.
\end{abstract}

Keywords: Brain like, Nervous system injury and repair, Flexible neural network, Key group generation sequence, High-dimensional information field,Memory analysis

\section{INTRODUCTION}

Flexible depth neural network (KDNN) with unipolar and multipolar flexible weakly nonlinear clustering functions with parameters is designed,and it is that corresponding learning algorithm. Different from the ordinary neighborhood depth neural network (KDNN), KDNN can not only learn the connection weight, but also learn the parameters of flexible weakly nonlinear clustering function. Therefore, it can generate appropriate weak nonlinear clustering function morphology for each hidden layer and output layer unit according to the learning sample set. Flexible neural network can improve the performance of kdnn network and solve the classification and prediction problems in different fields.

From non flexible depth neural network (KFDNN) to flexible depth neural network (KFDNN), and then from flexible depth neural network (KFDNN) to brain like neural network; The relationship between hypersection of brain like heavy nucleus boundary key group generation sequence, flexible depth neural network (KFDNN) and brain like neural network is constructed.

\subsection{Flexible neural network mathematical model}

$$
\begin{aligned}
& \forall K_{D N N}^{n-1}\left(\rho_{\wedge}^{n}, \theta^{\lambda}\right) \stackrel{\text { Iterations }}{\longrightarrow} \exists K_{D N N}^{n-1}\left(\rho_{\wedge}^{m}, \theta^{k} \otimes \beta^{k}\right), \text { if } \theta \otimes \beta, \rho \text { and appearing weak nonlinearity } \\
& S^{m+k-1}\left[\left(\rho^{m} \otimes \theta^{k}\right)^{+} \wedge\left(\rho^{m} \otimes \theta^{k}\right)^{-}\right] \stackrel{\text { Left,right hemisp heres }}{\text { (Superball ,Hypersp here })} \longrightarrow\left[S_{\text {left }}^{m+k-1}\left(\rho^{m} \otimes \theta^{k}\right)^{+}\right] \wedge\left[S_{\text {right }}^{m+k-1}\left(\rho^{m} \otimes \theta^{k}\right)^{-}\right]
\end{aligned}
$$

\subsection{Mathematical model of brain like neural network analysis}

Its core core is the left and right brain heavy nuclei of high-dimensional supersymmetric hypersurface normal complex variable high-dimensional tangent bundle.

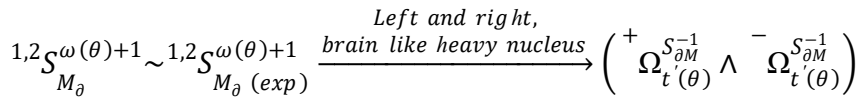


The left and right brain heavy nuclei are substituted into the mathematical model of flexible neural network,then

$$
S_{\text {left }}^{m+k-1}\left({ }^{+} \Omega_{t^{\prime}(\theta)}^{S_{\partial M}^{-1}}\right) \wedge S_{\text {right }}^{m+k-1}\left({ }^{-} \Omega_{t^{\prime}(\theta)}^{S_{\partial M}^{-1}}\right) \simeq S_{\text {Left }}^{m+k-1}\left({ }^{+} \Omega_{t^{\prime}(\theta)}^{S_{\partial M}^{-1}}\left(\rho^{t} \otimes \theta^{k}\right)\right) \wedge S_{\text {right }}^{m+k-1}\left({ }^{-} \Omega_{t^{\prime}(\beta)}^{S_{\partial M}^{-1}}\left(\rho^{t} \otimes \beta^{k}\right)\right)
$$

The relationship and evolution between the formation of neurons and $\mathrm{K}$ iterations.

$$
\begin{aligned}
& \text { if } \rho \rightarrow 1, \theta=2 k \pi+\theta_{1}+\theta_{2}+\cdots, t \in \forall \sigma,\left(S_{\partial M}^{-1}\right)^{k}, \text { then } \\
& \quad S_{\text {Left }}^{m+k-1}\left({ }^{+}{ }_{t^{\prime}(\theta)}^{\left(S_{\partial M}^{-1}\right)^{k}}\left(\theta^{k}\right)\right) \wedge S_{\text {right }}^{m+k-1}\left({ }^{-} \Omega_{t^{\prime}(\beta)}^{\left(S_{\partial M}^{-1}\right)^{k}}\left(\beta^{k}\right)\right) \cong S_{\text {Left,right }}^{m+k-1}\left({ }^{+} \Omega_{t^{\prime}(\theta \wedge \beta)}^{S_{\partial M}^{-1}}\left(\theta^{k} \otimes \beta^{k}\right)\right)
\end{aligned}
$$

The distribution of left and right hemispheres (brain like) is delayed. Perform a mathematical model to analysis of the effect.

The weak nonlinear fluctuation of information field is formed on the $t^{\prime}$ tangent disturbance of $\theta^{k}, \beta^{k}$. The internal law can be observed by combining the above formula.

i. The iterative hyper slice kernel and high-dimensional time tangent perturbation kernel are analyzed.

$$
\left[{ }^{ \pm} S_{\partial M}^{-k}\left(\theta^{k} \wedge \beta^{k}\right)\right],{ }^{ \pm} \Omega^{\prime}[t(\theta) \wedge t(\beta)]_{\partial}^{k}
$$

ii. The time tangent problem of the hypercut kernel of the left and right brain (brain like).

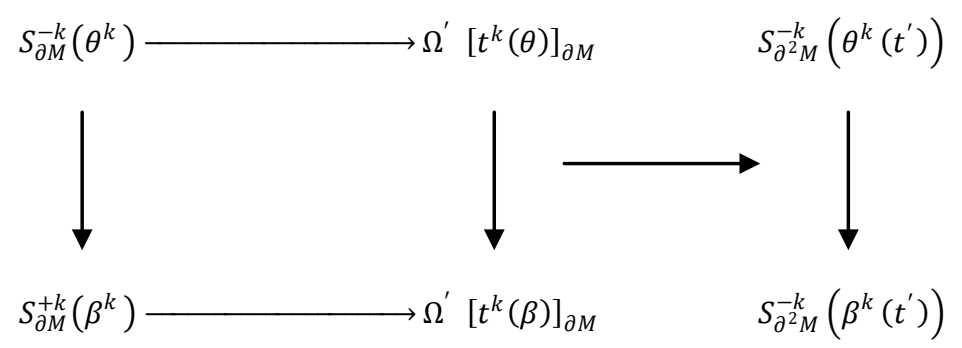

The time tangent perturbation structure of left and right brain (brain like) overweight nuclei is called neurons for short. Then ${ }_{\text {Left }} S_{\partial^{2} M}^{-k}\left(\theta^{k}\left(t^{\prime}\right)\right){ }_{\text {right }} S_{\partial^{2} M}^{-k}\left(\beta^{k}\left(t^{\prime}\right)\right)$. Therefore, neurons in the left and right brain (brain like) have different division of labor and operate in the dimension of time tangent, that is, information storage, operation, extraction, analysis and so on.

iii. How neurons are distributed in the sulcus of the left and right brain (brain like).

$$
{ }^{1,2} S_{M_{\partial}(\exp )}^{\omega(\theta)+1}\left[S_{\partial^{2} M}^{-k}\left(\theta^{k}\left(t^{\prime}\right)\right) \wedge S_{\partial^{2} M}^{-k}\left(\beta^{k}\left(t^{\prime}\right)\right)\right]
$$

That is, sulcus gyrus causes brain like distribution dimension +1 , Moreover, the distribution of neurons presents the morphological characteristics of cooperative operation of probability distribution. Therefore, the human brain has the reason of changeable and innovation. The repair of the injured nervous system of the local nerve of the human brain is similar to that of the brain like nervous system, that is, the loss of local data of memory leads to amnesia; But it will not cause dimensional disaster of high-dimensional information field, The lead of memory restoration, that is, the time tangent between neurons, links the information of all dimensions.

$$
{ }^{1,2} S_{M_{\partial}(\exp )}^{\omega(\theta)+1}\left[\left[S_{\partial^{2} M}^{-k}\left(\theta^{k}\left(t^{\prime}\right) \wedge \theta_{\partial}\left(t^{\prime}\right)\right)\right] \wedge\left[S_{\partial^{2} M}^{-k}\left(\beta^{k}\left(t^{\prime}\right) \wedge \beta_{\partial}\left(t^{\prime}\right)\right)\right]\right],
$$

Local missing function analysis of human brain (brain like) information data. 


$$
\begin{aligned}
& { }^{1,2} S_{M_{\partial}(\exp )}^{\omega(\theta)+1}{ }^{\partial}\left({ }_{\wedge} \theta_{\partial}\left(t^{\prime}\right)\right) \wedge^{1,2} S_{M_{\partial}(\exp )}^{\omega(\theta)+1}{ }^{\partial}\left({ }_{\wedge} \beta_{\partial}\left(t^{\prime}\right)\right) \sim^{1,2} S_{M_{\partial}(\exp )}^{\omega(\theta)+1}{ }^{\partial}\left({ }_{\wedge} \theta_{\partial}\left(t^{\prime}\right) \wedge{ }_{\wedge} \beta_{\partial}\left(t^{\prime}\right)\right) \\
& { }^{1,2} S_{M_{\partial}(\exp )}^{\omega(\theta)+1}\left({ }^{\partial} \theta_{\partial}\left(t^{\prime}\right) \wedge{ }_{\wedge} \beta_{\partial}\left(t^{\prime}\right)\right) \simeq{ }^{1,2} S_{M_{\partial}(\exp )}^{\omega(\theta)+1}{ }^{\partial}\left(\theta_{\partial}^{\prime}\left(t^{\prime}\right)\right), \text { then } \\
& \exists\left[\begin{array}{c}
1,2\left[\omega\left(\theta^{\prime}\right)+1\right] \\
\partial M_{\partial}(\exp )
\end{array}\left(\theta_{\partial^{2}}^{\prime}\left(t^{\prime}\right)\right)\right]_{\text {lost data }}^{\text {Drop 2 dimensions }}=\exists\left[\begin{array}{c}
1,2\left[\omega\left(\theta^{\prime}\right)+1\right] \\
M_{\partial}^{2}(\exp )
\end{array}\left(\theta_{\partial^{2}}^{\prime}\left(t^{\prime}\right)\right)\right] \\
& \text { if } t^{\prime} \rightarrow-\infty \text {, then , and non-existent data caused total amnesia. }
\end{aligned}
$$

iv. Neurons are accompanied by left and right brain damage (local), structural morphology.

$$
\begin{aligned}
& { }^{1,2} S_{M_{\partial}(\exp )}^{\omega(\theta)+1}\left[S_{\partial^{2} M}^{-k}\left(\theta^{k}\left(t^{\prime}\right)\right) \wedge S_{\partial^{2} M}^{-k}\left(\beta^{k}\left(t^{\prime}\right)\right)\right]-\left[\begin{array}{r}
1,2\left[\omega\left(\theta^{\prime}\right)+1\right] \\
M_{\partial}^{2}(\exp )
\end{array}\left(\theta_{\partial^{2}}^{\prime}\left(t^{\prime}\right)\right)\right]_{l o s t} \\
& \int\left[\begin{array}{c}
1,2[\omega(\theta)+1] \\
M_{\partial}(\exp )
\end{array}\left(\theta_{\partial}^{\prime}\left(t^{\prime}\right)\right)\right]_{l o s t} \text { Repair data from the perspective of mathematical model. }
\end{aligned}
$$

v. Data characteristics of neurons (left and right brain (brain like)) repairing local damage.

$$
\begin{gathered}
\int{ }^{1,2} S_{M_{\partial}(\exp )}^{\omega(\theta)+1}\left[S_{\partial M}^{-k}\left(\theta^{k}\left(t^{\prime}\right)\right) \wedge S_{\partial M}^{-k}\left(\beta^{k}\left(t^{\prime}\right)\right)\right]+\int\left[\begin{array}{r}
1,2\left[\omega\left(\theta^{\prime}\right)+1\right] \\
M_{\partial}(\exp )
\end{array}\left(\theta_{\partial}^{\prime}\left(t^{\prime}\right)\right)\right]_{\text {lost }} \\
=\sum^{1,2} S_{M_{\partial}(\exp )}^{\omega(\theta)+1}\left[S_{\partial M}^{-k}\left(\theta^{k}\left(t^{\prime}\right) \oplus \theta_{\partial}^{\prime}\left(t^{\prime}\right)\right) \wedge S_{\partial M}^{-k}\left(\beta^{k}\left(t^{\prime}\right) \oplus \theta_{\partial}^{\prime}\left(t^{\prime}\right)\right)\right] \\
{ }^{1,2} S_{M_{\partial}(\exp )}^{\omega(\theta)+1}\left[S_{\partial^{2} M}^{-k}\left(\theta^{k}\left(t^{\prime}\right)\right) \wedge S_{\partial^{2} M}^{-k}\left(\beta^{k}\left(t^{\prime}\right)\right)\right] \\
=\sum{ }^{1,2} S_{M_{\partial}(\exp )}^{\omega(\theta)+1}\left[S_{\partial M}^{-k}\left(\theta^{k}\left(t^{\prime}\right) \oplus \theta_{\partial}^{\prime}\left(t^{\prime}\right)\right) \wedge S_{\partial M}^{-k}\left(\beta^{k}\left(t^{\prime}\right) \oplus \theta_{\partial}^{\prime}\left(t^{\prime}\right)\right)\right]
\end{gathered}
$$

Therefore, the repair of human brain (brain like) damage is generally distributed and obtained in the time tangent angle, that is, the relationship between dimension reduction and dimension increase of data.At the same time, there is the relationship between partial differential and integral (local) $\sum \theta_{\partial}^{\prime}\left(t^{\prime}\right)$.

vi. The repair forms of local nerves in the left and right brain of human brain are different. Please observe the following formula.

$$
\left\{\begin{array}{c}
{ }^{+} \Omega_{M}^{\partial}\left(\theta^{k}\left(t^{\prime}\right) \oplus \theta_{\partial}^{\prime}\left(t^{\prime}\right)\right)_{\text {Left }} \\
-\Omega_{M}^{\partial}\left(\beta^{k}\left(t^{\prime}\right) \oplus \theta_{\partial}^{\prime}\left(t^{\prime}\right)\right)_{\text {right }}
\end{array}\right.
$$

Therefore, the left and right brain can cooperate to repair the local nervous system and restore the amnesia to normal.

$$
{ }^{\partial} \Omega_{M}^{k}\left[\theta^{k} \beta^{k}\left(t^{\prime}\right) \oplus \theta_{\partial}^{\prime}\left(t^{\prime}\right)\right]=\Omega_{M}^{k+1}[\theta(\rho(t))]
$$

Therefore, the coordination of left and right brain (brain like) can better develop the brain and is also conducive to the repair of brain injury.

$$
S_{\text {Left }}^{m+k-1}\left({ }^{+} \Omega_{t^{\prime}(\theta \wedge \beta)}^{\left(S_{\partial M}^{-1}\right)^{k}}\left(\theta^{k} \wedge \beta^{k}\right)\right) \cong \Omega_{M}^{k+1}[\theta(\rho(t))]_{S_{\text {left }, \text { right }}^{m+k-1}}
$$

\subsection{The Human brain (brain like) perceives the surrounding information field (assuming Similar MR} information). 


$$
\begin{aligned}
& \Omega^{k+1}[\theta(\rho(t(M R)))]=\Omega^{k+1}\left[\theta\left(\rho_{t}\left(\sum \frac{\delta}{\omega_{i}} \times \log \left|I+R^{-1} \times H_{i j} \times Q_{M R}^{\text {Core energy }} \times H_{j i}^{H}\right|\right)\right)\right] \text {, and } \\
& R^{-1} \text { interference signal, } Q_{M R}^{\text {Core energy }}=\operatorname{Matrix}\left[\begin{array}{lll}
E_{X_{E}}^{K} \otimes X_{K}^{H} & & \\
& E_{X_{S}}^{K} \otimes X_{K}^{H} & \\
& & E_{X_{M}}^{K} \otimes X_{K}^{H}
\end{array}\right]_{i}^{Q}
\end{aligned}
$$

i. The eye perception image of the human brain is equivalent to how signal of $M R^{H_{i j}} Q_{i} H_{j i}^{H} \quad$ is processed in brain space.

The energy wave structure equation of human brain (brain like) supporting information field.

$$
\left.\left.\Omega^{k+1}\left[\theta\left(\rho\left(t\left(Q_{M R}^{\text {Core energy }}\right)\right)\right)\right]=S_{\text {Left }}^{m+k-1}\left({ }^{+} \Omega_{t^{\prime}\left(\theta \wedge \beta\left(Q_{M R}^{-1}\right)^{k}\right.}^{(\text {core energy }}\right)\right)\left(\theta^{k} \wedge \beta^{k}\left(Q_{M R}^{\text {Core energy }}\right)\right)\right)
$$

ii. The vector motion of energy fluctuation on the brain space surface $\left(X_{K}^{H}\right)$, so the above formula can be written as.

$$
\begin{aligned}
& \Omega^{k+1}\left[\theta\left(\rho_{t}\left(\text { Matrix }\left[\begin{array}{lll}
E_{X_{E}}^{K} \otimes X_{K}^{H} & & \\
& E_{X_{S}}^{K} \otimes X_{K}^{H} & \\
& & E_{X_{M}}^{K} \otimes X_{K}^{H}
\end{array}\right]_{i}^{Q}\right)\right)\right]
\end{aligned}
$$

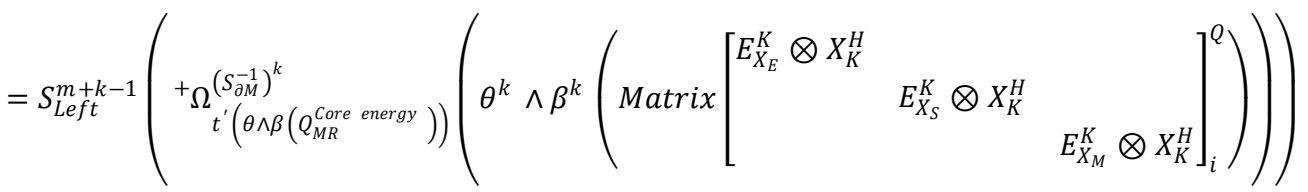

It can be seen from the above that the brain carries special energy waves and processes various signals in a higher dimension.

$$
\begin{gathered}
\Omega^{k+1}\left[\theta\left(\rho_{t}\left(\operatorname{Matrix}\left[\begin{array}{lll}
E_{X_{E}}^{K} \otimes X_{K}^{H} & & \\
& E_{X_{S}}^{K} \otimes X_{K}^{H} & \\
& & E_{X_{M}}^{K} \otimes X_{K}^{H}
\end{array}\right]_{i}^{Q}\right)\right)\right] \\
\left.\quad-\operatorname{Sin}\left(\frac{\beta_{1}}{2}+\frac{\pi}{4}+n \cdot \frac{\pi}{4}\right) \operatorname{Cos}\left(\sum_{i=2}^{m} \beta_{i}+\sum_{i=2}^{m} i \cdot \frac{\beta_{i}}{2}\right)\right]_{\theta \wedge \beta\left(t^{\prime}\right)}
\end{gathered}
$$

Using the image definition function kernel of MR and integrating the right structure of the above formula, we can observe the polar coordinate image with higher dimension. And reference to Figure 1.

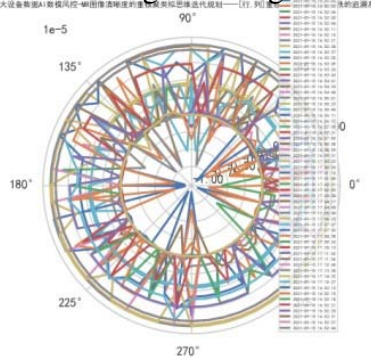

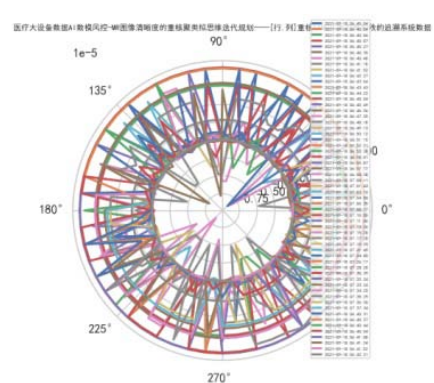

Figure 1. Higher dimensional MR image sharpness kernel function polar image. 
iii. Perceive the fluctuation regular of MR information field in a higher dimension. $\omega_{i}$ is the angular velocity $\left(\omega_{i}=T R \otimes\right.$ $T E$ ) High frequency wave angular velocity: $\omega_{i}\left(\delta^{-1}\right)$,High frequency wave carrying image information.

$$
\begin{aligned}
& \omega_{i}^{-1}(\delta) \times \log \left(H_{i j} \times Q_{M R}^{\text {Core energy }} \times H_{j i}^{H}\right)=\frac{\log \left(H_{i j} \times Q_{M R}^{\text {Core energy }} \times H_{j i}^{H}\right)}{\omega_{i}(\delta)} \\
& \frac{\theta}{\rho(t)}=\frac{\log \left(H_{i j} \times Q_{M R}^{\text {Core energy }} \times H_{j i}^{H}\right)}{\omega_{i}(\delta)}, \quad \theta \times \omega_{i}(\delta)=\rho(t) \times \log \left(H_{i j} \times Q_{M R}^{\text {Core energy }} \times H_{j i}^{H}\right) \\
& \Omega^{k+1}\left(\theta \cdot \rho_{t}\left(Q_{M R}^{\text {Core energy }}\right)\right) \rightarrow \frac{1}{(k+1) k(k-1) \ldots} \times S_{\text {Left,right }}^{m+k-1}\left(\theta_{t^{\prime}}^{k}\right)_{\rho \rightarrow \delta} \\
& \theta=\frac{\rho(t)}{\omega_{i}(\delta)} \times \log \left(H_{i j} \times Q_{M R}^{\text {Core energy }} \times H_{j i}^{H}\right) \text { is substituted into the above formula, then } \\
& \frac{1}{(k+1) k(k-1) \ldots} \times S_{\text {Left }, \text { right }}^{m+k-1}\left(\frac{\rho(t)}{\omega_{i}(\delta)} \times \log \left(H_{i j} \times Q_{M R}^{\text {Core energy }} \times H_{j i}^{H}\right)\right)_{\partial}^{k} \text {, and if } m \rightarrow 0, t^{\prime}, \text { then } \\
& {\left[{ }_{\text {Left }} S_{\partial^{2} M}^{-k}\left(\theta^{k}\left(t^{\prime}\right)\right) \wedge_{\text {right }} S_{\partial^{2} M}^{-k}\left(\beta^{k}\left(t^{\prime}\right)\right)\right]} \\
& =\frac{1}{(k+1) k(k-1) \ldots} \times S_{\text {Left }, \text { right }}^{m+k-1}\left(\frac{\rho(t)}{\omega_{i}(\delta)} \times \log \left(H_{i j} \times Q_{M R}^{\text {Core energy }} \times H_{j i}^{H}\right)\right)_{\partial}^{k}
\end{aligned}
$$

Equation (9) above is the functional equation of the dimensionality reduction process of high-frequency wave carrying image information field.

vi. KFDNN has a dimension reduction process (gradient descent) in neural network training and learning.

If $\omega_{i}(\delta)$ high-frequency wave and brain like (human brain) wave have some low-frequency co vibration resonance, it will make the human brain uncomfortable, that is

$$
\begin{aligned}
& \frac{(k+1) k(k-1) \ldots}{\omega_{i}\left(\delta^{-1}\right)} \times\left(S^{-k+1}\right), \rightarrow \frac{\omega_{i}(\delta)}{(k+1) k(k-1) \ldots} \times S_{\text {Left,right }}^{k-1}\left(Q_{M R}^{\text {Core energy }}\right) \\
& \frac{\omega_{i}(T R \otimes T E)}{(k+1) k(k-1) \ldots} \times S_{\text {Left }, \text { right }}^{k-1}\left(Q_{M R}^{\text {Core energy }}\right)_{\delta}^{H}
\end{aligned}
$$

The above function structure is the form of low-frequency co vibration resonance wave carrying image information.

$$
\begin{aligned}
& S_{\text {Left,right }}^{-k+1}\left(Q_{M R}^{\text {Core energy }}\right)_{\delta}^{H}=\frac{\omega_{i}^{-1}(T R \otimes T E)}{(k+1) k(k-1) \ldots} \times\left[\operatorname{Cos}\left(\sum_{i=2}^{m} \theta_{i}+\sum_{i=2}^{m} i \cdot \frac{\theta_{i}}{2}\right)-\operatorname{Cos}\left(\sum_{i=2}^{m} \beta_{i}+\sum_{i=2}^{m} i \cdot \frac{\beta_{i}}{2}\right)\right], \\
& \text { and } \delta \rightarrow 1 \text {, or } \delta \rightarrow-\infty \\
& S_{\text {Left right }}^{-k+1}\left(Q_{M R}^{\text {Core energy }}\right)_{\delta}^{H}=\frac{\left(\frac{1}{4}\right)^{n}}{(k+1) k(k-1) \ldots} \times\left[\operatorname{Sin}\left(\theta_{i}+\sum_{i=2}^{m} \theta_{i}+n \cdot \frac{\pi}{4}\right)+\operatorname{Sin}\left(\theta_{i}-\sum_{i=2}^{m} \theta_{i}+n \cdot \frac{\pi}{4}\right)\right], \\
& \frac{(k+1)+k(k-1)+\cdots}{\left(\frac{1}{4}\right)^{n} \times(k+1) k(k-1) \ldots \times \omega_{i}(T R \otimes T E)}=\frac{\left[\operatorname{Sin}\left(\theta_{i}+\sum_{i=2}^{m} \theta_{i}+n \cdot \frac{\pi}{4}\right)-\operatorname{Sin}\left(\theta_{i}-\sum_{i=2}^{m} \theta_{i}+n \cdot \frac{\pi}{4}\right)\right]}{\left[\operatorname{Cos}\left(\sum_{i=2}^{m} \theta_{i}+\sum_{i=2}^{m} i \cdot \frac{\theta_{i}}{2}\right)-\operatorname{Cos}\left(\sum_{i=2}^{m} \beta_{i}+\sum_{i=2}^{m} i \cdot \frac{\beta_{i}}{2}\right)\right]}
\end{aligned}
$$




$$
\begin{gathered}
\frac{(k+1)+k(k-1)+\cdots}{\omega_{i}(T R \otimes T E)} \simeq \frac{\left[\operatorname{Sin}\left(\theta_{i}+\sum_{i=2}^{m} \theta_{i}+n \cdot \frac{\pi}{4}\right)+\operatorname{Sin}\left(\theta_{i}-\sum_{i=2}^{m} \theta_{i}+n \cdot \frac{\pi}{4}\right)\right]}{\left[\operatorname{Cos}\left(\sum_{i=2}^{m} \theta_{i}+\sum_{i=2}^{m} i \cdot \frac{\theta_{i}}{2}\right)-\operatorname{Cos}\left(\sum_{i=2}^{m} \beta_{i}+\sum_{i=2}^{m} i \cdot \frac{\beta_{i}}{2}\right)\right]} \\
\frac{1}{\omega_{i}(T R \otimes T E)} \stackrel{\text { reduction }}{\longrightarrow} \frac{\left[\operatorname{Sin}\left(\theta_{i}+\sum_{i=2}^{m} \theta_{i}+n \cdot \frac{\pi}{4}\right)+\operatorname{Sin}\left(\theta_{i}-\sum_{i=2}^{m} \theta_{i}+n \cdot \frac{\pi}{4}\right)\right]}{\lambda_{i}\left[\operatorname{Cos}\left(\theta_{i}+\sum_{i=2}^{m} \theta_{i}\right)-\operatorname{Sin}\left(\theta_{i}-\sum_{i=2}^{m} \theta_{i}+n \cdot \frac{\pi}{4}\right)\right]} \times \operatorname{tg}\left(\sum \theta_{i}\right) \\
\frac{1}{\omega_{i}(T R \otimes T E)}=\operatorname{ctg}\left(\sum_{i=2}^{m} i \cdot \frac{\theta_{i}}{2}\right), \therefore \\
S_{\text {Left } \text { right }}^{-k+1}\left(Q_{M R}^{\text {Core energy }}\right)_{\delta}^{H}=\operatorname{ctg}\left(\sum_{i=2}^{m} i \cdot \frac{\theta_{i}}{2}\right)_{E_{X}}
\end{gathered}
$$

The following and reference to Figure 2. (formula (11)) shows the brain like (left and right brain of human brain) of the three-dimensional image of the reduced resonance wave morphological equation of low-frequency co vibration carrying image information. $S_{\text {Left, right }}^{-k+1}\left(Q_{M R}^{\text {Core energy }}\right)_{\delta}^{H}$
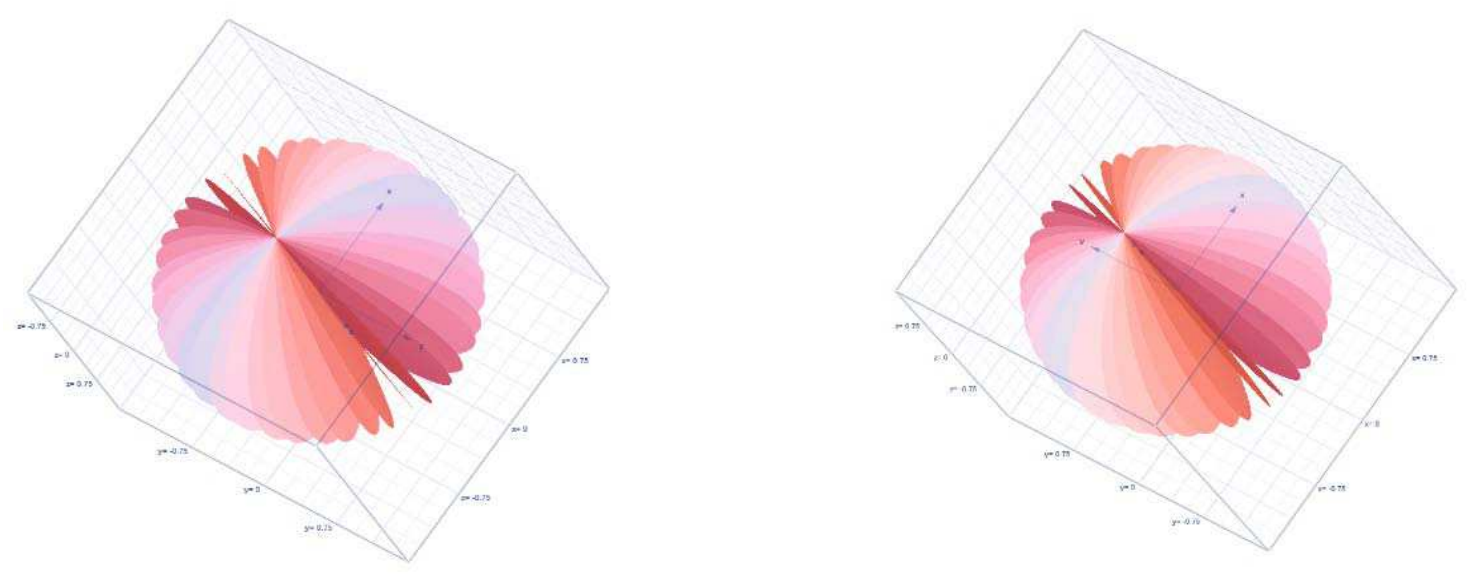

Figure 2. Three dimensional image of reduced resonance wave morphological equation of brain like with image information.

The left and right brain (brain like) kernels cooperate with the transformation of the fitting equation carrying the information reduced wave form.

$$
{ }^{+} \Omega_{t^{\prime}(\theta \wedge \beta)}^{\left(S_{\partial M}^{-1}\right)^{k}}\left(\theta^{k} \wedge \beta^{k}\right) \sim \sum_{k \geq 3}^{m} S_{\text {Left }, \text { right }}^{-k+1}\left(Q_{M R}^{\text {Core energy }}\right)_{\delta}^{H}, \text { if } S_{\text {Left }, \text { right }}^{-k+1} \subset \operatorname{ctg}\left(\sum_{i=2}^{m} i \cdot \frac{\theta_{i}}{2}\right)_{E_{X}}
$$

Each reduced $S^{-1}$-slice stores a large amount of information, including information fragments similar to MR images. On the whole, it is a high-dimensional data of massive information stored in the brain-like(human brain). And there is a higher dimension information of the key group to extract the information, which is called the distribution table group of high dimension information. It is equivalent to the generation sequence of key group, so equation (5) is simplified to

$$
S_{\text {Left }, \text { right }}^{m+k-1}\left(\sum_{k \geq 3}^{m} S_{\text {Left, right }}^{-k+1}\left(Q_{M R}^{\text {Core energy }}\right)_{\delta}^{H}\right) \sim S_{\text {Left }, \text { right }}^{m+k-1}\left(\sum_{k \geq 3}^{m} \operatorname{ctg} s\left(\sum_{i=2}^{m} i \cdot \frac{\theta_{i}}{2}\right)_{E_{X\left(t^{\prime}\right)}}^{Q_{M R}}\right) \text {, and s is expressed dimension }
$$




$$
S_{\text {Left }, \text { right }}^{m+k-1}\left(\sum_{k \geq 3}^{m} S_{\text {Left, right }}^{-k+1}\left(Q_{M R}^{\text {Core energy }}\right)_{\delta}^{H}\right) \sim S_{\text {Left, right }}^{m+k-1}\left(\sum_{k \geq 3}^{m} c t g^{s}\left(\sum_{\rho=2}^{m} \rho_{\theta} \cdot \frac{\theta_{\rho\left(t^{\prime}\right)}}{2}\right)_{\left.E_{X\left(t^{\prime}\right.}\right)}^{Q_{M R}}\right) \text {, and }
$$

$s \geq 3$ is expressed dimension, $\rho\left(t^{\prime}\right)$ is the polar diameter of polar coordinates, $t^{\prime}$ is the time tangent

The key group generates the sequence $\left({ }^{+} \Omega_{t^{\prime}(\theta)}^{S_{\partial M}^{-1}} \wedge{ }^{-} \Omega_{t^{\prime}(\theta)}^{S_{M}}\right)$ to the left and right brain (brain like) kernels, and cooperates with and carries the information to reduce the fluctuation fitting transformation (formula (13)). A large amount of information (such as MR image information) is stored on each reduced $S^{-1}$, and the extraction of information requires the generation sequence of key group, i.e. distribution table group (guidance), which may have a cotangent timeline $\rho_{\theta}\left(t^{\prime}\right)$.
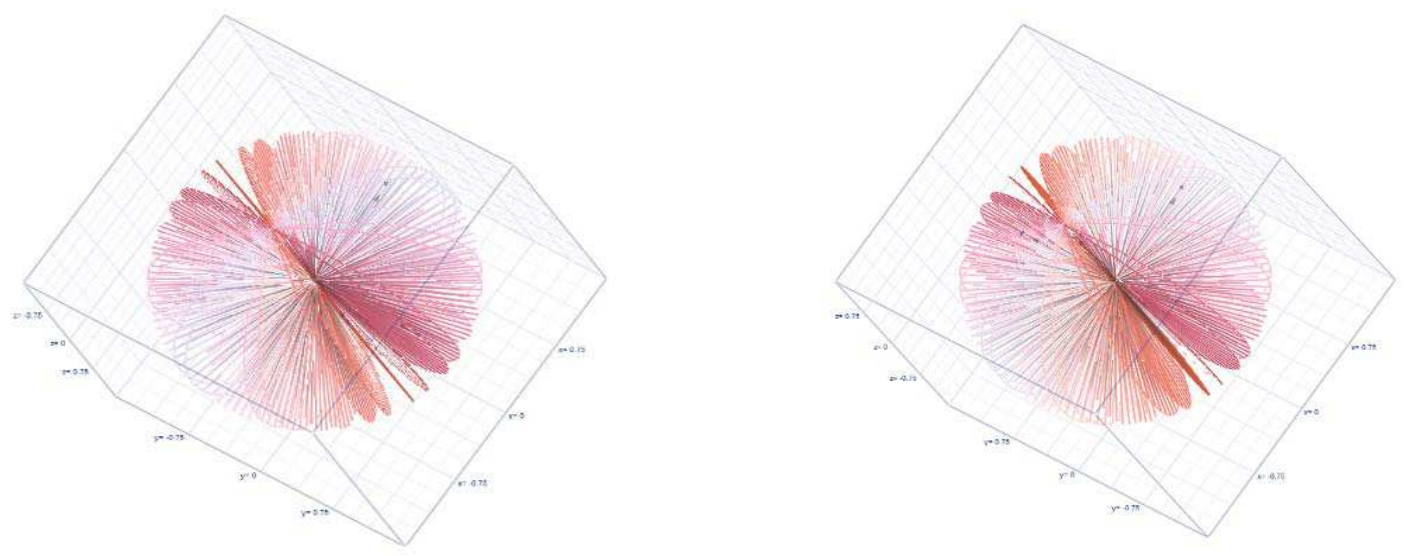

Figure 3. The generation sequence of the key group of each piece of $S^{-1}$ information has a cotangent time line $\rho_{\theta}\left(t^{\prime}\right)$.

In the high-dimensional information field, there is a hidden timeline $\rho_{\theta}\left(t^{\prime}\right)$, and reference to Figure 3. that is, Cotangent bundle. It crosses the high-dimensional and low-dimensional brain like hypersections and $S_{k}^{-1}$-slice bundles, so it can be found that there may be the generation sequence of key groups in both brain like and human brain, and $\rho_{\theta}\left(t^{\prime}\right)$ cotangent bundle and $S_{k}^{-1}$ slice bundle.

$$
\begin{aligned}
& S_{\text {Left }, \text { right }}^{m+k-1}\left[\sum _ { k \geq 3 } ^ { m } \operatorname { c t g } ^ { s } \left(\sum_{\rho=2}^{m} \rho_{\theta}\left(\operatorname{Matrix}\left[\begin{array}{lll}
E_{X_{E}}^{K} \otimes X_{K}^{H} & \\
& E_{X_{S}}^{K} \otimes X_{K}^{H} & \\
& & E_{X_{M}}^{K} \otimes X_{K}^{H}
\end{array}\right]_{i}^{Q}\right)\right.\right. \\
& \left.\left.\cdot \frac{\theta_{\rho\left(t^{\prime}\right)}}{2}\right)_{E_{X\left(t^{\prime}\right)}}^{Q_{M R}}\right] \sim S_{\text {Left,right }}^{m+k-1}\left(\sum_{k \geq 3}^{m} S_{\text {Left,right }}^{-k+1}\left(Q_{M R}^{\text {Core energy }}\right)_{\delta}^{H}\right) \text {, and } s \text { is expressed dimension }
\end{aligned}
$$

$Q_{M R}^{\text {Core energy }}$ is the core energy to maintain brain like (human brain) memory (information storage medium) [i.e. memory suspension maintenance energy]. So $S_{k}^{-1}\left(Q_{M R}^{\text {Core energy }}\right)$-cut bundle (carrying energy), $\rho_{\theta}\left(t^{\prime}\left(Q_{M R}^{\text {Core energy }}\right)\right)$-cotangent bundle (carrying energy). $S_{k}^{-1}\left(Q_{M R}^{\text {Core energy }}\right)$-slice bundle carries a large amount of identifiable information data, which is suitable for brain like (human brain). The key group generation sequence of cotangent bundle $\rho_{\theta}\left(t^{\prime}\right)$ is used to extract useful information data, i.e

$$
S_{k}^{-1}\left(\rho_{\theta}\left(t^{\prime}\right)\right) \stackrel{\text { Extract data }}{\longrightarrow}\left[{ }^{+} \Omega_{t^{\prime}(\theta)}^{S_{\partial M}^{-1}} \wedge{ }^{-} \Omega_{t^{\prime}(\theta)}^{S_{\partial M}^{-1}}\right]
$$


${ }^{+} \Omega_{t^{\prime}(\theta)}^{S_{\partial M}^{-1}}\left(S_{k}^{-1}\left(\rho_{\theta}\left(t^{\prime}\right)\right)\right) \wedge{ }^{-} \Omega_{t^{\prime}(\theta)}^{S^{-1}}\left(S_{k}^{-1}\left(\rho_{\theta}\left(t^{\prime}\right)\right)\right)$ is the extraction information data function of the sequence generated by the key group.

\section{RECONSTRUCTED BRAIN LIKE NEURAL NETWORK R-KFDNN}

R-KFDNN Neuron structure function: ${ }^{+} \Omega_{t^{\prime}(\theta)}^{S_{\partial M}^{-1}}\left(S_{k}^{-1}\left(\rho_{\theta}\left(t^{\prime}\right)\right)\right) \wedge^{-} \Omega_{t^{\prime}(\theta)}^{S_{\partial M}^{-1}}\left(S_{k}^{-1}\left(\rho_{\theta}\left(t^{\prime}\right)\right)\right)$

R-KFDNN Neuron linked neural network: $\sum_{k \geq 3}^{m} \operatorname{ctg}^{s}\left(\sum_{\rho=2}^{m} \rho_{\theta} \cdot \frac{\theta_{\rho\left(t^{\prime}\right)}}{2}\right)_{E_{X\left(t^{\prime}\right)}}^{Q_{M R}}$

Therefore, the functional structure of brain like neural network is reconstructed:

$$
{ }^{+} \Omega_{t^{\prime}(\theta)}^{S_{\partial M}^{-1}}\left(S_{k}^{-1}\left(\sum_{k \geq 3}^{m} \operatorname{ctg} s\left(\sum_{\rho=2}^{m} \rho_{\theta} \cdot \frac{\theta_{\rho\left(t^{\prime}\right)}}{2}\right)^{Q_{E}}\right)\right) \wedge{ }^{-} \Omega_{t^{\prime}(\theta)}^{S_{\partial M}^{-1}}\left(S_{k}^{-1}\left(\sum_{k \geq 3}^{m} \operatorname{ctg} s\left(\sum_{\rho=2}^{m} \rho_{\theta} \cdot \frac{\theta_{\rho\left(t^{\prime}\right)}}{2}\right)^{Q_{E}}\right)\right)
$$

The above formula is the function body of local reconstruction brain like neural network of left and right brain (human brain). The following formula is the complex high-dimensional equation R-KFDNN that reconstructs the function body of the brain like (human brain) overall neural network.

$$
\begin{aligned}
& S_{\text {Left }, \text { right }}^{m+k-1}\left[\Omega_{t^{\prime}(\theta)}^{S_{M}^{-1}}\left(S_{k}^{-1}\left(\sum_{k \geq 3}^{m} \operatorname{ctg} s\left(\sum_{\rho=2}^{m} \rho_{\theta} \cdot \frac{\theta_{\rho(t)}}{2}\right)\right)\right]=\Omega^{Q_{E}+1}[\theta(\rho(t))]_{S_{\text {Left }, \text { right }}^{m+k-1} \text {, and } Q_{E}}\right. \\
& =\operatorname{Matrix}\left[\begin{array}{lll}
E_{X_{E}}^{K} \otimes X_{K}^{H} & & \\
& E_{X_{S}}^{K} \otimes X_{K}^{H} & \\
& & E_{X_{M}}^{K} \otimes X_{K}^{H}
\end{array}\right]_{i}^{Q} \text { is expressed core energy }
\end{aligned}
$$

The compact correlation between special flexible neural network and reconstructed brain neural network is established to solve the complexity problem in AI, and the hidden layer of KFDNN deep neural network. Slice bundle $S_{k}^{-1}$ equivalent to the key group generation sequence of brain like R-KFDNN, i.e

$$
+\Omega_{t^{\prime}(\theta)}^{S_{\partial M}^{-1}}\left(S_{k}^{-1}\left(\rho_{\theta}\left(t^{\prime}\right)\right)\right) \wedge{ }^{-} \Omega_{t^{\prime}(\theta)}^{-1}\left(S_{k}^{-1}\left(\rho_{\theta}\left(t^{\prime}\right)\right)\right) \text { is the hidden layer equivalent to KFDNN }
$$

The quasi thinking iterative programming of KFDNN is simpler and more practical than R-KFDNN in AI mathematical model. And KFDNN uses 3 sets of core formulas of depth statistics.

$$
\begin{aligned}
P_{\left(A_{i}, A_{j}\right)}^{(1)}=\left(\frac{1}{4}\right)^{n} \times & {\left[\operatorname{Sin}\left(A_{1}+\sum_{i=2}^{m} A_{i}+n \cdot \frac{\pi}{4}\right)+\operatorname{Sin}\left(A_{1}-\sum_{i=2}^{m} A_{i}+n \cdot \frac{\pi}{4}\right)\right]_{P_{i(x, y)}^{*}} } \\
P_{(A, B)}^{(2)}=\left(\frac{1}{4}\right)^{n-1} \times & \sqrt{2}\left[\operatorname{Sin}\left(\frac{A_{1}}{2}+\frac{\pi}{4}+n \cdot \frac{\pi}{4}\right) \operatorname{Cos}\left(\sum_{i=2}^{m} A_{i}+\sum_{i=2}^{m} i \cdot \frac{A_{i}}{2}\right)\right. \\
& \left.-\operatorname{Sin}\left(\frac{B_{1}}{2}+\frac{\pi}{4}+n \cdot \frac{\pi}{4}\right) \operatorname{Cos}\left(\sum_{i=2}^{m} B_{i}+\sum_{i=2}^{m} i \cdot \frac{B_{i}}{2}\right)\right]_{P_{i j\left(x_{i}, y_{j}\right)}^{*}}
\end{aligned}
$$


$[\operatorname{Tanh} \times \mathrm{Ctanh}]^{\nabla}$

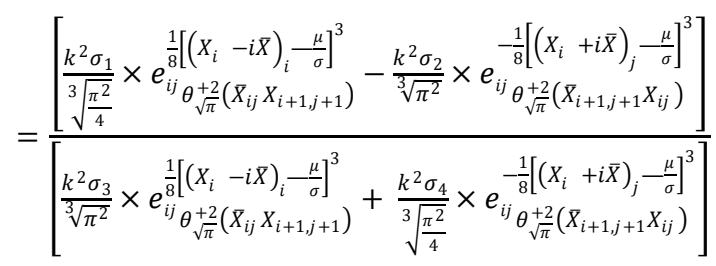

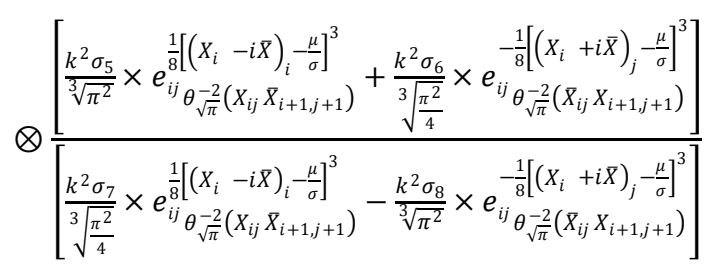

$$
\begin{aligned}
& \text {, and } \sigma\left(\pi, \frac{\pi}{4}, \frac{\pi}{2}, 2 \pi\right)^{-\mathrm{T}^{2}} \rightarrow \sigma\left(\pi, \frac{\pi}{4}, \frac{\pi}{2}, 2 \pi\right)^{\mathrm{T}^{2}} \text {, }
\end{aligned}
$$

i. KFDNN is trained and learned by KNN neural network, which greatly improves the risk control accuracy of AI mathematical model.

The reconstructed brain neuron network R-KFDDN is much more difficult than the above KFDNN. Its mathematical model itself has nonlinear disturbance in high-dimensional space, and the information field data processing runs on different levels, different dimensions, different tangent bundles and cotangent bundles. Key group is needed for data extraction. There is a hidden time tangent in data guidance, which is similar to data distribution table, but more complex than it.

Key group: ${ }^{+} \Omega_{t^{\prime}(\theta)}^{S_{\partial M}^{-1}}\left(S_{k}^{-1}\left(\rho_{\theta}\left(t^{\prime}\right)\right)\right) \wedge{ }^{-} \Omega_{t^{\prime}(\theta)}^{S_{\partial M}^{-1}}\left(S_{k}^{-1}\left(\rho_{\theta}\left(t^{\prime}\right)\right)\right)$

Brain like high dimensional morphology: $\Omega^{k+1}[\theta(\rho(t))]_{S_{\text {Left }, \text { right }}^{m+k-1}, \text { Different dimensions }}$

Different layer forms: $S_{\text {Left }, \text { right }}^{m+k-1}\left(\sum_{k \geq 3}^{m} \operatorname{ctg} g^{S}\left(\sum_{\rho=2}^{m} \rho_{\theta} \cdot \frac{\theta_{\rho\left(t^{\prime}\right)}}{2}\right)_{E_{X\left(t^{\prime}\right)}}^{Q_{M R}}\right)$

Different cut bundle morphology (slice bundle): $S_{k}^{-1}\left(\rho_{\theta}\left(t^{\prime}\right)\right)^{Q_{E}}$

Cotangent bundle form: $\rho_{\theta}\left(t^{\prime}\left(Q_{M R}^{\text {Core energy }}\right)\right)$

Data guided hidden time tangent: $\rho_{\theta}\left(t^{\prime}\left(Q_{E}\right)\right) \rightarrow \sum_{k \geq 3}^{m} \operatorname{ctg} s\left(\sum_{\rho=2}^{m} \rho_{\theta} \cdot \frac{\theta^{\rho(t)}}{2}\right)_{E_{X(t)}}^{Q_{M R}}$ is similar to the data allocation table, but more complex.

The key group is distributed on the slice bundle, i.e ${ }^{+} \Omega_{t^{\prime}(\theta)}^{S_{\partial M}^{-1}}\left(S_{k}^{-1}\right) \wedge{ }^{-} \Omega_{t^{\prime}(\theta)}^{S_{\partial M}^{-1}}\left(S_{k}^{-1}\right)$, and $S_{k}^{-1}$ is expressed slice bundle.

$$
{ }^{+\Lambda-} \Omega_{t^{\prime}(\theta)}^{S_{\partial M}^{-1}}\left(S_{k}^{-1}\left(\rho_{\theta}\left(t^{\prime}\right)\right)\right) \rightarrow \rho_{\theta}\left(t^{\prime}\right) \subset \sum_{k \geq 3}^{m} \operatorname{ctg} s\left(\sum_{\rho=2}^{m} \rho \cdot \frac{\theta_{\rho\left(t^{\prime}\right)}}{2}\right)_{E_{X\left(t^{\prime}\right)}}^{Q_{E}}
$$

That is, the key group should finally be distributed on the $\rho_{\theta}\left(t^{\prime}\right)$-Time tangent arc of $\sum_{k \geq 3}^{m} \operatorname{ctg} s\left(\sum_{\rho=2}^{m} \rho \cdot \frac{\left.\rho^{\rho(t}\right)}{2}\right)_{E_{X\left(t^{\prime}\right)}}^{Q_{E}}$. 
Sometimes in the brain like (human brain), the key group may be called the memory fragment distribution table.

ii. Memory analysis and AI mathematical model analysis of brain

$$
\begin{aligned}
& \omega^{s}\left(\lambda^{i}\right) \rightarrow{ }^{+\Lambda-} \Omega_{t^{\prime}(\theta)}^{S_{M}^{-1}}\left(S_{k}^{-1}\left(\rho_{\theta}\left(t^{\prime}\right)\right)\right), \text { and } \lambda^{i} \text { is brain wave like frequency, } \\
& \omega \text { is the angular velocity and } s \text { is the dimension } \\
& {\left[{ }^{+\wedge-} \Omega_{t^{\prime}(\theta)}^{S_{\partial M}^{-1}}\right]_{\rho_{\theta}}^{T} \sim \Omega^{s+1}\left(\frac{\omega^{s}\left(\lambda^{i}\right)}{S_{k}^{-1}\left(\rho_{\theta}\left(t^{\prime}\right)\right)}\right)}
\end{aligned}
$$

Introduction to memory analysis - mirror reflection (with local random data loss)

$$
\left[\begin{array}{ccc}
\omega^{s}\left(\lambda^{i}\right) & S_{k}^{-1}\left(\rho_{\theta}\left(t^{\prime}\right)\right) \\
& { }^{s} \Omega^{T}\left(\omega, S_{k}^{-1}\right) & \\
\rho_{\theta}\left(t^{\prime}\right) & & Q_{E}
\end{array}\right] \sim\left[{ }^{+\wedge-} \Omega_{t^{\prime}(\theta)}^{S_{M}^{-1}}\right]{ }^{T}
$$

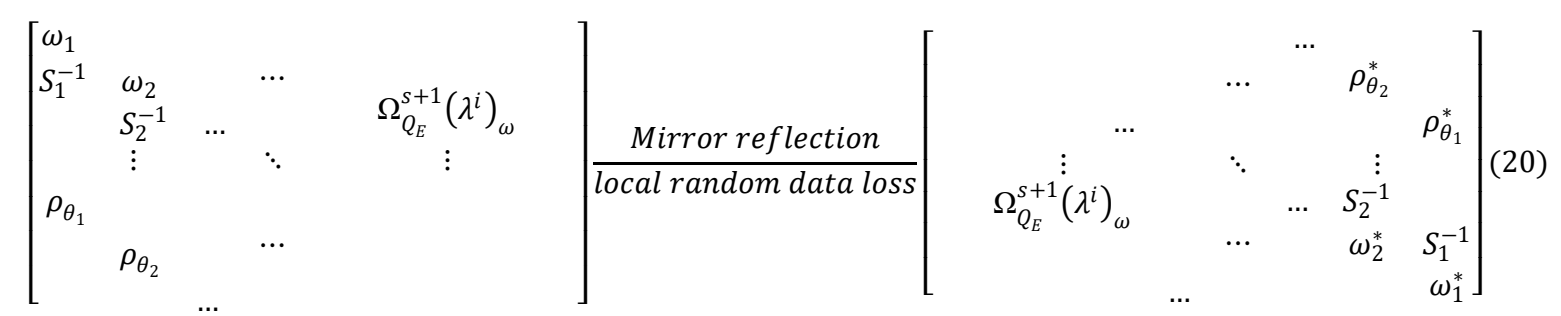

if ${ }^{+} \Omega_{Q_{E}}^{s+1}\left(\lambda^{i}\right)_{\omega} \vee{ }^{-} \Omega_{Q_{E}}^{s+1}\left(\lambda_{*}^{i}\right)_{\omega} \cong I^{s+1}\left(\lambda_{*}^{i}\right)_{\omega}$, and $s$ is the dimension, $\omega$ is the amplitude, $\lambda^{i}$ or $\lambda_{*}^{i}$ is the frequency.

Therefore, the key variable of memory analysis is $I^{s+1}\left(\lambda_{*}^{i}\right)_{\omega}$, which obtains brain like (human brain) information data through the mirror reflection of high-dimensional information field.

$$
\left[{ }^{+\wedge-} \Omega_{t^{\prime}(\theta)}^{S_{\partial M}^{-1}}\right]_{\rho_{\theta}}^{T} \rightarrow\left[{ }^{+} \Omega_{Q_{E}}^{S+1}\left(\lambda^{i}\right)_{\omega} \vee{ }^{-} \Omega_{Q_{E}}^{s+1}\left(\lambda_{*}^{i}\right)_{\omega}\right]
$$

Parsing is embedded in information and runs on a higher dimension. Memory parsing requires high speed $\omega^{s}\left(\lambda^{i}\right)$, and linear.

$$
\begin{gathered}
\text { Memory Parsing: } I_{\text {pass }}^{s+1}\left(\lambda_{*}^{i}\right)_{\omega}:\left[{ }^{+} \Omega_{Q_{E}}^{s+1}\left(\lambda^{i}\right)_{\omega} \vee{ }^{-} \Omega_{Q_{E}}^{s+1}\left(\lambda_{*}^{i}\right)_{\omega}\right]_{\rho_{\theta}(t)}^{S_{k}^{-1}} \\
I_{\text {pass }}^{s+1}\left(\lambda_{*}^{i}\right)_{\omega}:\left[{ }^{+} \Omega_{Q_{E}}^{s+1}\left(\lambda^{i}\right)_{\omega} \vee{ }^{-} \Omega_{Q_{E}}^{s+1}\left(\lambda_{*}^{i}\right)_{\omega}\right]_{\rho_{\theta}\left(t^{\prime}\right)}^{S_{k}^{-1}} \\
I_{\text {pass }}^{3}\left(\lambda_{*}^{i}\right)_{\omega}:\left[{ }^{+} \Omega_{Q_{E}}^{3}\left(\lambda^{i}\right)_{\omega} \vee{ }^{-} \Omega_{Q_{E}}^{3}\left(\lambda_{*}^{i}\right)_{\omega}\right]_{\rho_{\theta}\left(t^{\prime}\right)}^{S_{k}^{-1}} \\
I_{\text {pass }}^{2}\left(\lambda_{*}^{i}\right)_{\omega}:\left[{ }^{+} \Omega_{Q_{E}}^{2}\left(\lambda^{i}\right)_{\omega} \vee{ }^{-} \Omega_{Q_{E}}^{2}\left(\lambda_{*}^{i}\right)_{\omega}\right]_{\rho_{\theta}\left(t^{\prime}\right)}^{S_{k}^{-1}}
\end{gathered}
$$

The compact compression of $\rho_{\theta}\left(t^{\prime}\right)$, that is, the compression structure with time $t^{\prime}$ at the same time. $I_{\text {pass }}^{s+1}\left(\lambda_{*}^{i}\right)_{\omega}$ will switch freely in the high-dimensional information field. Therefore, the key to memory analysis is in $I_{\text {pass }}^{s+1}\left(\lambda_{*}^{i}\right)_{\omega\left(t^{\prime}\right)}$, which 
is a linear wave structure with special frequency.

\section{CONCLUSION}

Reconstruct the brain like neural network R-KFDNN, generate the sequence super tangent plane from the brain like heavy nucleus boundary key group for the first time, and fuse with the flexible depth neural network (KFDNN) and brain like neural network.From the perspective of nervous system repair of local nerve damage, this paper analyzes how the brain obtains memory analysis from the distribution table group of Time tangent bundle with fingerprint feature key group generation sequence, and so as to provide useful help for memory recovery.

\section{REFERENCES}

[1] Zhu RongRong, Differential Incremental Equilibrium Theory, Fudan University,Vol 1, 2007:1-213

[2] Zhu RongRong, Differential Incremental Equilibrium Theory, Fudan University,Vol 2, 2008:1-352

[3] Liu zhuanghu, Simplicity Set Theory, Beijing China ,Peking University Press, 2001.11: 1-310

[4] Xie bangjie, Transfinite Number and Theory of Transfinite Number, Jilin China, jilin people's publishing house, 1979.01:1-140

[5] Nan chaoxun, Set Valued Mapping, Anhui China, Anhui University Press , 2003.04: 1-199

[6] Li hongyan, On some Compactness and Separability in Fuzzy Topology, Chengdu China,,Southwest Jiaotong University Press, 2015.06: 1-150

[7] Bao zhiqiang, An introduction to Point Set Topology and Algebraic Topology, Beijing China, Peking University Press, 2013.09:1-284

[8] Gao hongya, Zhu yuming, Quasiregular Mapping and A-harmonic Equation, Beijing China, Science Press, 2013.03: $1-218$

[9] C. Rogers W. K. Schief, Bäcklund and Darboux Transformations: Geometry and Modern Applications in Solition Theory, first published by Cambridge University, 2015: 1-292.

[10]Ding Peizhu,Wang Yi, Group and its Express, Higher Education Press, 1999: 1-468.

[11]Gong Sheng, Harmonic Analysis on Typical Groups Monographs on pure mathematics and Applied Mathematics Number twelfth, Beijing China, Science Press, 1983: 1-314.

[12] Gu chaohao, Hu Hesheng, Zhou Zixiang, DarBoux Transformation in Solition Theory and Its Geometric Applications (The second edition), Shanghai science and technology Press, 1999, 2005: 1-271.

[13] Jari Kaipio Erkki Somersalo, Statistical and Computational Inverse Problems With 102 Figures, Spinger.

[14] Numerical Treatment of Multi-Scale Problems Porceedings of the 13th GAMM-Seminar, Kiel, January 24-26, 1997 Notes on Numerical Fluid Mechanics Volume 70 Edited By WolfGang HackBusch and Gabriel Wittum.

[15] Qiu Chengtong, Sun Licha, Differential Geometry Monographs on pure mathematics and Applied Mathematics Number eighteenth, Beijing China, Science Press, 1988: 1-403.

[16] Ren Fuyao, Complex Analytic Dynamic System, Shanghai China, Fudan University Press, 1996: 1-364.

[17] Su Jingcun, Topology of Manifold, Wuhan China, wuhan university press, 2005: 1-708.

[18] W. Miller, Symmetry Group and Its Application, Beijing China, Science Press, 1981: 1-486.

[19] Wu Chuanxi, Li Guanghan, Submanifold geometry, Beijing China, Science Press, 2002: 1-217.

[20] Xiao Gang, Fibrosis of Algebraic Surfaces, Shanghai China, Shanghai science and technology Press, 1992: 1-180.

[21] Zhang Wenxiu, Qiu Guofang, Uncertain Decision Making Based on Rough Sets, Beijing China, tsinghua university press, 2005: 1-255. 
[22] Zheng jianhua, Meromorphic Functional Dynamics System, Beijing China, tsinghua university press, 2006: 1-413.

[23] Zheng Weiwei, Complex Variable Function and Integral Transform, Northwest Industrial University Press, 2011: 1-396. 


\section{Supplementary Files}

This is a list of supplementary files associated with this preprint. Click to download.

- HigherdimensionalMRimagesharpnesskernelfunctionpolarimage01.jpg 